\title{
Diagnosis of myocardial infarction following hospitalisation for exacerbation of COPD
}

\author{
David A. McAllister*,\#, John D. Maclay*, Nicholas L. Mills ${ }^{\star}$, Andrew Leitch ${ }^{+}$, \\ Philip Reid ${ }^{\S}$, Ross Carruthers ${ }^{f}$, Jennifer O'Connor $^{f}$, Lawrence McAlpine ${ }^{\S}$, \\ George Chalmers ${ }^{+}$, David E. Newby ${ }^{\uparrow}$, Elaine Clark**, Peter W. Macfarlane** \\ and William MacNee*
}

ABSTRACT: Cardiovascular disease is common in chronic obstructive pulmonary disease (COPD) and raised troponin is common in exacerbations. However, the prevalence of myocardial infarction following hospitalisation for exacerbation of COPD is unknown.

Patients aged $\geqslant 40$ yrs hospitalised with acute exacerbation of COPD $(n=242)$ with $\geqslant 10$ packyrs of cigarette smoking were included in a prospective case series conducted in four hospitals. Patients whose primary presenting complaint was chest pain or who had an alternative diagnosis were excluded. Chest pain histories, serial ECGs and troponin levels were obtained.

The mean \pm SD age was $69 \pm 9$ yrs; 108 (45\%) patients were male and almost half were current smokers. 124 (51\%; 95\% Cl 48-58\%) patients had chest pain, which was exertional in 62 (26\%). 24 (10\%) had raised troponin, among whom, 20 (8.3\%; 95\% Cl 5.1-12.5\%) had chest pain and/or serial ECG changes, fulfilling the 2007 Universal Definition of Myocardial Infarction. Neither chest pain $(p=0.77)$ nor serial ECG changes $(p=0.39)$ were associated with raised troponin.

Raised troponin, chest pain and serial ECG changes are common in patients admitted to hospital with exacerbation of COPD. Overall, one in 12 patients met the criteria for myocardial infarction. Whether these patients would benefit from further cardiac investigation is unknown.

KEYWORDS: Chest pain, chronic obstructive pulmonary disease, ECG, myocardial infarction, troponin

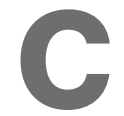

oronary heart disease is a major cause of mortality in chronic obstructive pulmonary disease (COPD) [1, 2]. Both conditions share common risk factors, such as smoking and socioeconomic status, and reduced forced expiratory volume in $1 \mathrm{~s}(\mathrm{FEV} 1)$, a characteristic feature of COPD, is an independent risk factor for cardiovascular mortality [3].

In studies using routine clinical data, diagnosis with myocardial infarction (MI) appears to be more common following exacerbations in patients with COPD $[4,5]$, while raised troponin has been found to be associated with increased mortality in exacerbation of COPD [6-8],

Coronary events may present without chest pain [9], can be missed even in simple chest pain presentations [10] and may be more likely to be missed in patients presenting with acute exacerbation of COPD who also report acute breathlessness and chest tightness.
Raised troponin is common in patients admitted to hospital with exacerbation of COPD [6-8]; however, we are unaware of any prospective study that has investigated this group for clinical or electrocardiography features of MI.

Therefore, we undertook a prospective case series to identify the prevalence of MI in patients admitted to hospital with acute exacerbation of COPD.

\section{METHODS}

Patients were recruited at four hospitals in central Scotland, UK: the Royal Infirmary of Edinburgh, Glasgow Royal Infirmary, Monklands Hospital (Airdrie) and Crosshouse Hospital (Kilmarnock). At each site, the local investigator recruited patients from emergency medicine departments and acute medical (admission) units. Patients aged $\geqslant 40 \mathrm{yrs}$, diagnosed with an acute exacerbation of COPD by a physician accredited in respiratory and/or general medicine and with $\geqslant 10$ pack-yrs of cigarette smoking were recruited. Patients were excluded if

\section{AFFILIATIONS}

*ELEGI/COLT Laboratories, Queens Medial Research Institute, University of Edinburgh,

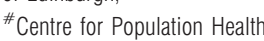
Sciences, University of Edinburgh, - Centre for Cardiovascular Science, University of Edinburgh, Edinburgh, +Respiratory Medicine, Glasgow Royal Infirmary,

**ECG Core Laboratory, Glasgow Royal Infirmary, University of Glasgow, Glasgow,

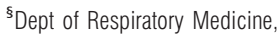
Monklands Hospital, NHS Lanarkshire, Airdrie, and ${ }^{f}$ Crosshouse Hospital, NHS Ayrshire and Arran, Kilmarnock, UK.

\section{CORRESPONDENCE}

W. MacNee

ELEGI/COLT Laboratories

Queens Medial Research Institute

University of Edinburgh

47 Little France Crescent

Edinburgh

EH16 4TJ

UK

E-mail: w.macnee@ed.ac.uk

Received:

July 212011

Accepted after revision:

Sept 022011

First published online:

Feb 092012 
chest pain was the primary presenting complaint, $>48 \mathrm{~h}$ had elapsed since admission, an alternative diagnosis was suggested by the admission chest radiograph or pulmonary embolism (PE) was confirmed on computed tomography pulmonary angiogram.

In the 2007 Universal Definition statement, myocardial infarction was defined as a rise and/or fall of troponin concentration together with evidence of myocardial ischaemia (at least one of the following: symptoms of ischaemia; new ST-T changes; new left bundle branch block; or development of pathological $Q$ waves in the ECG) [11]. We report the prevalence of MI diagnosed according to this definition, which does not imply a causal mechanism, and includes two subtypes: type $1 \mathrm{MI}$ is due to a primary coronary event, such as plaque rupture, and type 2 MI is secondary to increased myocardial oxygen demand or decreased supply.

A detailed chest pain and exacerbation history was obtained and recorded on a standard form. If an admission ECG had been performed, then this was obtained. If not, it was performed by the investigator, provided this could be done within $24 \mathrm{~h}$ of admission. The ECG was repeated between 12 and $36 \mathrm{~h}$ after the initial ECG. A nonfasting blood sample was taken and serum troponin, high-density lipoprotein and total cholesterol, triglycerides, full blood count and C-reactive protein were measured at each hospital laboratory. Where patients reported chest pain before or during the first $48 \mathrm{~h}$ of admission, the sample was taken $12 \mathrm{~h}$ after the onset of pain. If no chest pain was reported, troponin was obtained between 12 and $48 \mathrm{~h}$ following admission. Any chest pain or raised troponin developing after $\geqslant 48 \mathrm{~h}$ following admission would not be identified in this study.

Spirometry results were either obtained from the patient's case notes or from the local pulmonary function service. Research ethics committee approval was obtained from Scottish Multicentre Research Ethics Committee A (Edinburgh, UK), and patient consent was obtained for all study protocols and procedures (06/MRE10/78).

Troponin I (ARCHITECT; Abbott Diagnostics, Abbott Park, IL, USA) was measured at the Royal Infirmary of Edinburgh and Glasgow Royal Infirmary and troponin $\mathrm{T}$ (Roche, Basel, Switzerland) was measured at Monklands and Crosshouse Hospitals. The Universal Definition of Myocardial Infarction recommends the use of the 99th percentile of the normal reference population to define raised troponin and stipulates that the assay should have a co-efficient of variation of $\leqslant 10 \%$ $(10 \% \mathrm{CV})$ at the 99th percentile value [11]. However, until recently, no troponin assay could achieve this level of precision. We followed the recommendation of APPLE et al. [12], and defined the cut-off as the lowest concentration measurable with $<10 \%$ coefficient of variation. To facilitate reporting of results from multiple centres, we have expressed troponin concentrations in multiples of the $10 \%$ CV level at each site [12].

All ECGs were coded using the Minnesota code, the most widely used ECG coding system, which is used to assign codes to pathological and variant features in 12-lead ECGs in research studies [13]. A single reader (D.A. McAllister) coded all ECGs, blinded to all clinical features, blood results and the order of ECG recordings. In 48 ECGs collected at the Glasgow site, Minnesota codes were also reported electronically [14]. Agreement for electronic and paper reads for the presence/absence of Q-waves,
ST depression and $\mathrm{T}$ wave flattening/inversion was calculated $(\kappa=0.73,0.61$ and 0.57 , respectively). Serial ECG changes indicative of an acute cardiac event were defined using the Minnesota code criteria for independently read serial ECGs [13].

Although the occurrence of MI in the absence of chest pain is well documented [9], certain features of the chest pain history are recognised as being high-risk or more indicative of myocardial ischaemia (pain that is "like a pressure", radiates to either shoulder or arm, or is related to exertion) while other features are considered low-risk (pleuritic, related to movement, reproduced by palpation, sharp or of very short or long duration $(<2 \mathrm{~min}$ or $>12 \mathrm{~h}$ )) [15]. On this basis, patients were classified as having either low- or high-risk chest pain. Patients with neither low- nor high-risk features were categorised as indeterminate.

Each exacerbation was categorised as probably infectious or otherwise using the criteria of ANTHONISEN et al. [16] (type 1: three major infectious symptoms (increased sputum purulence, sputum volume and dyspnoea): type 2: two major infectious symptoms: type 3: one major infectious symptoms, plus additional minor symptoms) [16].

The prevalence of MI was calculated along with 95\% confidence intervals using the exact binomial test. In exploratory analyses, associations between patient characteristics and raised troponin concentrations were examined using Fisher's exact test, for which we report all associations where the $\mathrm{p}$-value was $<0.10$. Analyses were performed using SAS version 9.2 (SAS Institute, Cary, NC, USA) and R version 2.11.1 (The R Group, Vienna, Austria).

\section{RESULTS}

A total of 242 patients were recruited: 143 from the Royal Infirmary of Edinburgh, 48 from Glasgow Royal Infirmary, 42 from Monklands Hospital and nine from Crosshouse Hospital. The mean \pm SD age was $69 \pm 9 \mathrm{yrs} ; 108$ (45\%) patients were male and almost half were current smokers, with the majority having severe or very severe airflow limitation on spirometry (table 1). All patients were breathless on admission, increased sputum volume was present in $130(54 \%)$ and sputum purulence in 141 $(58 \%)$, leukocytosis in $113(47 \%)$, and raised C-reactive protein $\left(>6 \mathrm{mg} \cdot \mathrm{dL}^{-1}\right)$ in $158(74 \%)$. All patients were treated with nebulised bronchodilators, $217(90 \%)$ with oral prednisolone and $164(68 \%)$ with antibiotics. The median (interquartile range) length of stay was 5 (3-8) days and $21(15 \%)$ out of the 143 patients at the Edinburgh site (where follow-up was available via electronic records) died within 12 months of admission.

$24(10 \%)$ patients had elevated serum troponin, with $22(9 \%)$ patients having a level greater than twice the cut-off (defined as the $10 \% \mathrm{CV}$ level for the assay) and $15(6 \%)$ having a serum troponin level greater than three times the cut-off.

$20(8.3 \%$; 95\% CI $5.1-12.5 \%)$ patients met the criteria for the Universal Definition for Myocardial Infarction (fig. 1) [11] as they had raised troponin along with ECG changes (new Q waves, or serial changes in T-waves or ST segments) and/or chest pain. Six (2.5\%; 95\% CI 1.0-5.6\%) patients had raised troponin, chest pain and serial ST-T wave ECG changes.

Overall, chest pain was common, with 124 (51\%; 95\% CI 48-58\%) patients reporting chest pain, which was most commonly tight or sharp, but in 50 patients ( $40 \%$ of those with chest pain) the pain was high-risk for myocardial ischaemia (table 2). 


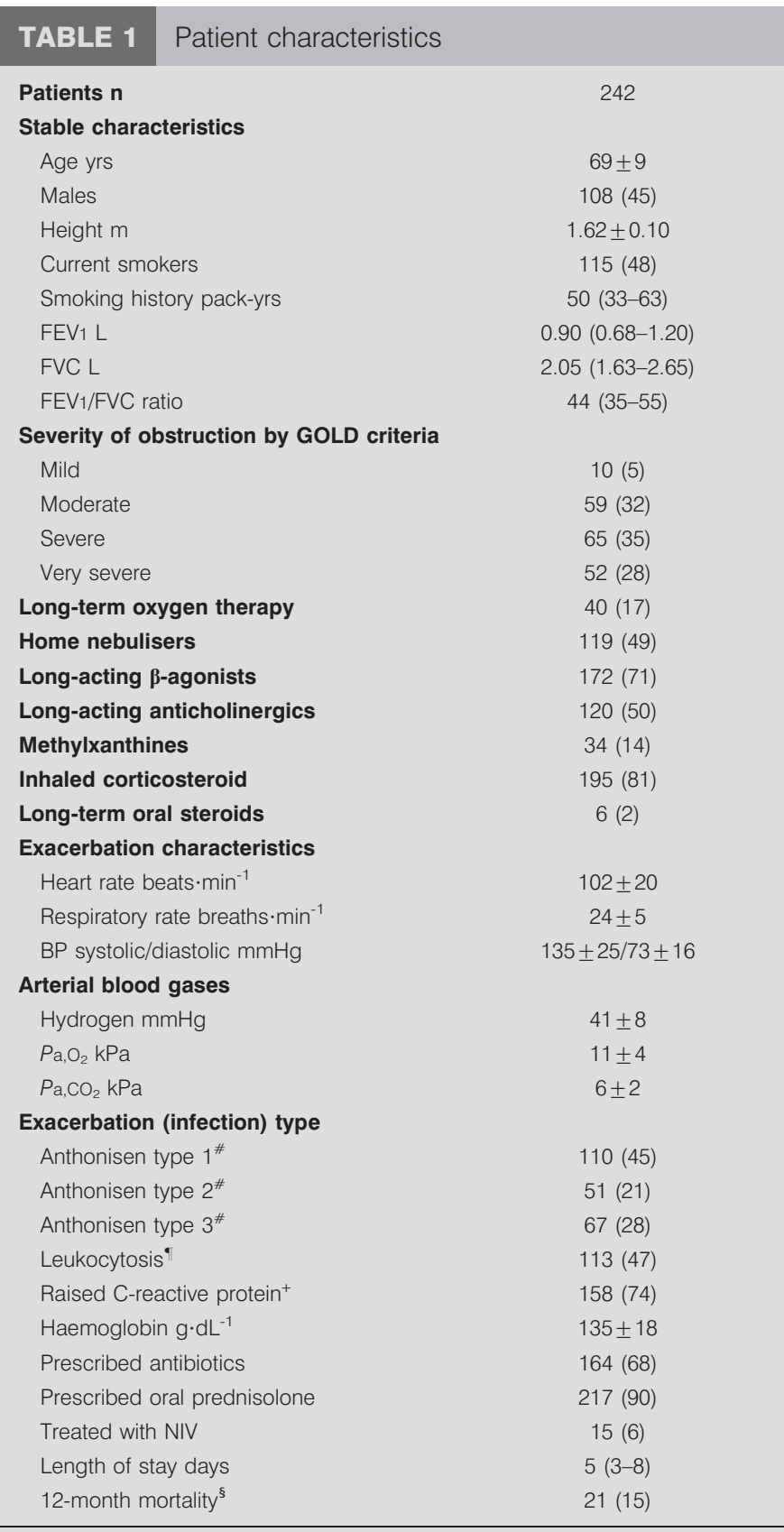

Data are presented as mean $\pm \mathrm{SD}, \mathrm{n}(\%)$ or median (interquartile range), unless otherwise stated. FEV1: forced expiratory volume in $1 \mathrm{~s}$; FVC: forced vital capacity; GOLD: Global Initiative for Chronic Obstructive Lung Disease; BP: blood pressure; $\mathrm{Pa}, \mathrm{O}_{2}$ : arterial oxygen tension; $\mathrm{Pa}_{1} \mathrm{CO}_{2}$ : arterial carbon dioxide tension; NIV: noninvasive ventilation. ${ }^{\#}$ : according to the criteria of ANTHONISEN et al. [16]; ' : white cell count $>11 \times 10^{9}$ cells $\cdot \mathrm{L}^{-1} ;{ }^{+}:>6 \mathrm{mg} \cdot \mathrm{dL}^{-1}$; ${ }^{\text {s: }}$. Royal Infirmary of Edinburgh (Edinburgh, UK).

Sinus tachycardia was the commonest abnormality on admission ECG and was found in $112(46 \%)$ patients. $14(6 \%)$ patients had atrial fibrillation, $15(6 \%)$ had right bundle branch block and $12(5 \%)$ had left bundle branch block. $20(8 \%)$ had $\mathrm{P}$ pulmonale. Q waves considered "diagnostic" in the Minnesota coding system and $\mathrm{T}$ wave inversion were both common (34 $(14 \%)$ and $37(15 \%)$ respectively).

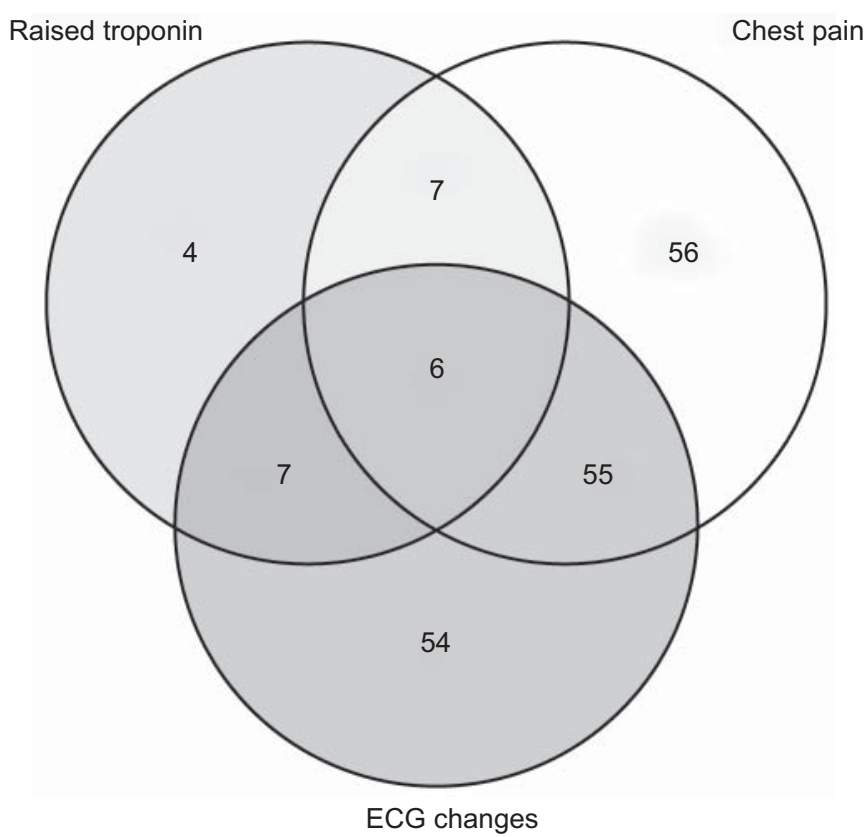

FIGURE 1. Number of patients with chest pain, serial ECG changes and raised troponin.

$18(7.5 \%)$ patients had no ECG within the first $24 \mathrm{~h}$ of admission, while $21(8.6 \%)$ had no second ECG. Only three patients developed $\mathrm{Q}$ waves during admission, but serial changes in $\mathrm{T}$ wave inversion/flattening and ST depression were common (65 (32\%) and $19(9 \%)$, respectively). $13(6 \%)$ had serial changes in ST elevation (online supplementary material).

Neither any chest pain nor high-risk chest pain was associated with raised troponin $(p=0.77$ and $p=0.29$, respectively). Raised troponin may be more common in patients with admission ECGs with Minnesota codes for MI $(p=0.06)$ but there was no evidence that serial ECG changes were more common $(p=0.39$; table 3$)$.

Cardiovascular risk factors were common (table 4). Over 30\% of patients had a past medical history of ischaemic heart disease, 34 $(14 \%)$ had previous stroke or peripheral vascular disease and 101 $(42 \%)$ had vascular disease at any site. Hypertension and hypercholesterolaemia were also common. Amongst the 141 patients without known vascular disease, 19 (13\%) had an estimated (Framingham) risk of a cardiac event within $10 \mathrm{yrs}$ of $\geqslant 20 \%$ [17]. Around one-third of patients were on regular statin therapy and 96 (40\%) were on antiplatelet therapy, although only five $(2 \%)$ were on a $\beta$-blocker.

In exploratory analyses, we examined the associations between raised troponin and a wide range of patient characteristics, including severity of airflow obstruction, performance status, smoking status, usual and acute therapy, anaemia, raised inflammatory markers and symptoms suggestive of infection, and arterial blood gases. There was a suggestion that diabetes, right bundle branch block, acidosis and methylxanthine therapy in patients with exacerbation of COPD may be associated with raised troponin.

Interestingly, the use of a long-acting $\beta$-agonist was less common in patients with raised troponin (table 5). 


\begin{tabular}{lc} 
TABLE 2 Chest pain characteristics & \\
Central & $76(61)$ \\
Duration $<\mathbf{2}$ min or $>\mathbf{1 2} \mathbf{~ h}^{\#}$ & $19(18)$ \\
Radiated to arm or jaw" & $13(11)$ \\
Character & \\
Tight & $60(48)$ \\
Sharp\# & $24(19)$ \\
Dull & $12(10)$ \\
Like a pressure & $11(9)$ \\
Heavy & $4(3)$ \\
Burning & $1(2)$ \\
Gripping & $2(2)$ \\
Autonomic symptoms, volunteered & $8(6)$ \\
Precipitating factors & \\
Exertion & $62(52)$ \\
Exertion, but also present at rest & $44(36)$ \\
Deep inspiration & \\
Coughing & $7(6)$ \\
Palpation, pain reproduced & \\
Relieving factors, partial or complete & $20(16)$ \\
Nitrate therapy & $11(9)$ \\
Bronchodilator therapy & \\
Oxygen therapy & $18(15)$ \\
Had similar pain previously & $15(12)$ \\
During an exacerbation & $13(10)$ \\
When stable & \\
Summary of chest pain & $67(54)$ \\
Lowh-risk features only & $31(25)$ \\
Low- and high-risk features & \\
Neither low- nor high-risk features & $50(40)$ \\
\hline & $21(17)$ \\
& $22(18)$ \\
& $31(25)$ \\
\hline
\end{tabular}

Data are presented as $n(\%) .{ }^{\#}$ : low risk of myocardial ischaemia; ${ }^{\circ}$ : high risk of myocardial ischaemia.

\section{DISCUSSION}

Cardiovascular disease is common in patients with COPD [1, 2]. In a prospective case series in patients admitted to hospital with acute exacerbation of COPD, we found that approximately one in 12 patients had raised troponin along with serial ECG changes and/or chest pain, meeting the criteria of the Universal Definition for Myocardial Infarction [11].

Two previous prospective studies report the prevalence of raised troponin in exacerbation of COPD. In one consecutive case series of 71 patients admitted to an intensive care department with acute exacerbation of COPD, 13 (18\%; 95\% CI 11-29\%) had raised troponin [6], and in a larger consecutive series of 250 patients with exacerbation of COPD admitted to a single centre, $17 \%$ had raised troponin [8]. In a study using routine data where troponin was measured in 441 out of 996 patients admitted with exacerbation of COPD at the discretion of the treating clinician, troponin was raised in $27 \%$ (12\% overall) [7]. Our findings suggest that most of these patients with raised troponin also have chest pain and/or serial ECG changes, and so meet the criteria for the Universal Definition for Myocardial Infarction [11].

A substantial proportion of these myocardial infarctions are likely to have developed secondary to increased myocardial

\begin{tabular}{|c|c|c|c|c|}
\hline \multirow[t]{2}{*}{ TABLE 3} & \multirow[b]{2}{*}{$>3 \times$ cut-off $^{\#}$} & \multirow[b]{2}{*}{$>$ cut-off $^{\#}$} & \multirow[b]{2}{*}{$\leqslant$ cut-off $^{\#}$} & \multirow[b]{2}{*}{ p-value } \\
\hline & & & & \\
\hline Patients n & 15 & 9 & 218 & \\
\hline Any chest pain & $9(60)$ & $4(44)$ & $111(51)$ & 0.77 \\
\hline High-risk chest pain & $2(13)$ & 0 & $48(22)$ & 0.29 \\
\hline \multicolumn{5}{|l|}{ First-admission ECG } \\
\hline Diagnostic ECG & $4(27)$ & $3(33)$ & $28(13)$ & 0.06 \\
\hline Equivocal ECG & $5(33)$ & $3(33)$ & $80(36)$ & $>0.99$ \\
\hline Sinus tachycardia & $8(53)$ & $7(78)$ & $97(44)$ & 0.14 \\
\hline Left bundle block & 0 & 0 & $12(6)$ & $>0.99$ \\
\hline Right bundle block & $3(20)$ & 0 & $12(6)$ & 0.08 \\
\hline P pulmonale & $2(13)$ & $1(11)$ & $17(8)$ & 0.36 \\
\hline Right axis deviation & $2(13)$ & $1(11)$ & $17(8)$ & 0.36 \\
\hline Serial ECG changes & $7(50)$ & $4(50)$ & $72(40)$ & 0.39 \\
\hline
\end{tabular}

Data are presented as $\mathrm{n}(\%)$, unless otherwise stated. \#: cut-offs differed between sites and were based on the lowest concentration where the coefficient of variation was $<10 \%$ as recommended by the 2007 Universal Definition [11] as follows. Royal Infirmary of Edinburgh, Edinburgh, UK (ARCHITECT troponin I assay; Abbott Diagnostics, Abbott Park, IL, USA): $0.15 \mathrm{ng} \cdot \mathrm{mL}^{-1}$ prior to introduction of a new assay (November 17, 2007) and $0.05 \mathrm{ng} \cdot \mathrm{mL}^{-1}$ thereafter; Glasgow Royal Infirmary, Glasgow, UK (ARCHITECT): $0.04 \mathrm{ng} \cdot \mathrm{mL}^{-1}$; Monklands Hospital, Airdrie and Crosshouse Hospital, Kilmarnock, UK (troponin T assay; Roche, Basel, Switzerland): $0.03 \mathrm{ng} \cdot \mathrm{mL}^{-1}$

demand/reduced oxygen supply. Under the Universal Definition for Myocardial Infarction, such events, usually resulting in subendocardial rather than transmural infarction, are termed type $2 \mathrm{MI}$ [11]. Over half of our patients were tachycardic (with either sinus tachycardia or atrial fibrillation), which, together with hypoxaemia and increased afterload, is likely to increase myocardial oxygen demand and reduce supply. Similarly, in a previous study among 441 patients where troponin was measured at clinical discretion, raised troponin was also found to be associated with lower haemoglobin and tachycardia, favouring type $2 \mathrm{MI}$ [18].

During exacerbation of COPD, ischaemia secondary to increased afterload with consequent increased myocardial demand and reduced supply may also occur in the right heart, in a mechanism analogous to that proposed for the myocardial injury associated with acute PE [19]. Pulmonary hypertension is present in around a quarter of people with COPD awaiting lung transplant [20] with reversible increases in pulmonary artery pressure of $\sim 20 \mathrm{mmHg}$ having been described during acute respiratory failure in people with chronic bronchitis [21]. Such increases in pulmonary artery pressure may, in the context of tachycardia and underlying coronary disease, be sufficient to cause right ventricular ischaemia and an increase in troponin.

Around a fifth of patients were taking cardiac medications but only five $(2 \%)$ were taking $\beta$-blockers. Mortality has been found to be lower among patients with COPD taking $\beta$-blockers following admission to hospital with exacerbations [22] and during vascular surgery [23], and $\beta$-blocker prescription has recently been found to be associated with a lower risk of hospitalisation for exacerbation of COPD [24]. Prevention of 


\section{TABLE 4 Cardiovascular risk factors and therapy}

Patients $n$

IHD

None

Diagnosed by generalist

Diagnosed by specialist

Confirmed ${ }^{\#}$

Stroke or peripheral vascular disease

Hypertension

Hypercholesterolaemia

Diabetes

Family history of IHD

Creatinine $>120 \mu \mathrm{mol} \cdot \mathrm{L}^{-1}$

Nonfasting lipids

$\mathrm{HDL}$ cholesterol $\mathrm{mmol} \cdot \mathrm{L}^{-}$

Cholesterol/HDL ratio

Triglycerides $\mathrm{mmol} \cdot \mathrm{L}^{-1}$

Nitrate or nicorandil

Calcium channel blocker

$\beta$-blocker

ACE inhibitor/AR2 blocker

Diuretic

Statin therapy

Antiplatelet therapy

Antidiabetic therapy

Data are presented as $n(\%)$ or mean $\pm S D$, unless otherwise stated. IHD ischaemic heart disease; HDL: high-density lipoprotein; ACE: angiotensinconverting enzyme; AR: angiotensin receptor. ${ }^{*}$ : through diagnostic exercise tolerance testing, angiography or proven myocardial infarction.

tachycardia-related myocardial damage may be one mechanism underlying these associations. None of the five patients in our case series taking $\beta$-blockers had raised troponin, although this observation should be interpreted very cautiously as this association was not statistically significant.

However, myocardial ischaemia and MI following acute exacerbations might well arise due to plaque rupture and coronary thrombosis (type $1 \mathrm{MI}$ ). $50 \%$ of patients in our sample either had known vascular disease or an estimated 10-yr (Framingham) risk of a cardiac event of $\geqslant 20 \%$. Patients with COPD have an elevated risk of cardiovascular mortality [1, 2] and two previous observational studies using routine healthcare data found that the risk of diagnosis with MI was two-fold higher in patients with COPD during and immediately following acute exacerbation [4, 5]. Despite this, the use of secondary prevention was surprisingly low among our patients, with only a third receiving statins.

Inflammation has been implicated in plaque rupture [25], and exacerbation of COPD is associated with raised C-reactive protein, interleukin-6 and fibrinogen [26, 27]. Furthermore, we previously found that platelet activation was increased during exacerbations compared with the stable state [28], and an association between neutrophilia and raised troponin has been reported in acute exacerbation of COPD [18].

We believe that a proportion of patients with raised troponin in our case series were likely to have had a primary coronary event (type $1 \mathrm{MI})$. Two $(8 \%)$ patients out of the 24 with elevated

\begin{tabular}{|c|c|c|c|c|}
\hline \multirow[t]{2}{*}{ TABLE 5} & $\begin{array}{l}\text { troponin } r \\
\text { eristics }\end{array}$ & tive to $s$ & cted & \multirow[b]{2}{*}{ p-value } \\
\hline & $>3 \times$ cut-off $^{\#}$ & $>$ cut-off ${ }^{\#}$ & $\leqslant$ cut-off ${ }^{\#}$ & \\
\hline \multicolumn{5}{|l|}{ Airflow obstruction } \\
\hline Mild & $2(20)$ & $0(0)$ & $8(5)$ & \\
\hline Moderate & $2(20)$ & $1(20)$ & $56(33)$ & 0.19 \\
\hline Severe & $5(50)$ & $1(20)$ & $59(35)$ & \\
\hline Very severe & $1(10)$ & $3(60)$ & $48(28)$ & \\
\hline Long-term oxygen & $1(7)$ & $1(11)$ & $38(17)$ & 0.67 \\
\hline Current smokers & $7(47)$ & $3(33)$ & $105(48)$ & 0.71 \\
\hline Diabetes & $5(33)$ & $1(11)$ & $24(11)$ & 0.05 \\
\hline \multicolumn{5}{|l|}{ COPD therapy } \\
\hline LABAs & $7(47)$ & $5(56)$ & $160(73)$ & 0.05 \\
\hline Methylxanthines & $5(33)$ & $4(44)$ & $25(11)$ & 0.002 \\
\hline ICSs & $10(67)$ & 8 (89) & $177(81)$ & 0.34 \\
\hline \multicolumn{5}{|c|}{ Arterial blood gases } \\
\hline Acidosis & $5(33)$ & $3(33)$ & $33(15)$ & 0.04 \\
\hline Hypoxia & $3(20)$ & $1(11)$ & $75(34)$ & 0.24 \\
\hline Hypercapnia & $5(33)$ & $6(67)$ & $95(44)$ & 0.43 \\
\hline
\end{tabular}

Data are presented as $n(\%)$, unless otherwise stated. COPD: chronic obstructive pulmonary disease; LABA: long-acting $\beta$-agonist; ICS: inhaled

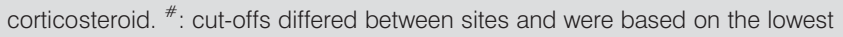
concentration where the coefficient of variation was $<10 \%$ as recommended by the 2007 Universal Definition [11] as follows. Royal Infirmary of Edinburgh, Edinburgh, UK (ARCHITECT troponin I assay; Abbott Diagnostics, Abbott Park, IL, USA): $0.15 \mathrm{ng} \cdot \mathrm{mL}^{-1}$ prior to introduction of a new assay (November 17 , 2007) and $0.05 \mathrm{ng} \cdot \mathrm{mL}^{-1}$ thereafter; Glasgow Royal Infirmary, Glasgow, UK (ARCHITECT): $0.04 \mathrm{ng} \cdot \mathrm{mL}^{-1}$; Monklands Hospital, Airdrie and Crosshouse Hospital, Kilmarnock, UK (troponin T assay; Roche, Basel, Switzerland): $0.03 \mathrm{ng} \cdot \mathrm{mL}^{-1}$.

troponin also had ST segment elevation or new Q waves, indicating transmural infarction, and may have benefited from coronary revascularisation. The overlap in symptoms between exacerbation of COPD and MI, and the absence of a simple diagnostic test to identify plaque rupture represent a major diagnostic challenge for clinicians. This problem is likely to become more pressing as increasingly sensitive troponin assays with lower thresholds for detection of troponin are introduced into clinical practice [29].

Risk stratification on the basis of chest pain history and serial ECGs is problematic for a number of reasons. Previous authors have suggested that central chest pain typical of MI is rare in exacerbation of COPD [5]; however, we found that central chest pain was common and patients commonly reported exertional chest pain prior to admission. There was no association between symptoms of chest pain and increased troponin concentrations, even when we restricted the analysis to those patients with symptoms that were considered to be suggestive of cardiac ischaemia [15]. The character of chest pain is of limited prognostic value in patients presenting with chest pain alone [30], and our findings suggest that it may be less useful still in patients with exacerbation of COPD.

Similarly, abnormalities were common on the resting admission ECG in patients with exacerbation of COPD, a finding which is 
consistent with previous reports that ECG abnormalities predictive of long-term risk of $\mathrm{MI}$ are common in patients hospitalised with acute exacerbation of COPD [31] and are associated with reduced FEV1 [32]. We found that changes in the $\mathrm{T}$ wave axis and dynamic ST segment depression on serial ECG testing were common, but were not associated with raised troponin. These changes may reflect transient myocardial ischaemia secondary to increased oxygen demand or reduced supply that is insufficient to induce MI or alternatively may be artifactual and nonspecific features of acute exacerbation. Consequently, it is not clear in which patients, if any, we should measure troponin where the primary clinical presentation is exacerbation of COPD.

Further observational studies are needed to better characterise which clinical features are associated with raised troponin and to determine the clinical significance of raised troponin in unselected patients with acute exacerbation of COPD, but randomised clinical trials of secondary prevention strategies are likely to be needed to address the diagnostic challenge of differentiating between primary acute coronary events and secondary myocardial ischaemia.

\section{Limitations}

This was not a consecutive sample of patients admitted with acute exacerbation of COPD. However, we did include patients from secondary care and tertiary referral centres, and our group was very similar, including in the prevalence of known ischaemic heart disease, to that described in the 2008 British Thoracic Society Audit of patients consecutively admitted to hospital with acute exacerbation of COPD (online supplementary material) [33].

Moreover, patients were not sampled on the basis of chest pain, troponin or ECG changes, and patients who presented to the treating clinician with evidence of MI, even if this followed a severe exacerbation of COPD, would not have been recruited to our study. Moreover, we report a lower proportion of patients with raised troponin than was found in previous studies [6-8]. As such, the true risk of $\mathrm{MI}$ in patients with severe exacerbation of COPD may be higher than suggested from our case series of patients hospitalised with exacerbation of COPD.

The troponin measurements were conducted in different laboratories using different assays rather than in a single centre. However, studying patients across multiple centres was a strength of the study, a recommended method for summarising the results across multicentre studies was employed and results were not directly compared between centres [12].

Raised troponin has been reported following PE in patients with normal coronary arteries on angiography [34], and although we did not include patients diagnosed with PE in our case series, we cannot exclude subclinical PE as a cause of the raised troponin. However, $\mathrm{PE}$ is found in only $3 \%$ of patients admitted to hospital with exacerbation of COPD [35], while we found raised troponin in three times that number, making it an unlikely explanation for raised troponin for the majority of patients.

The ECGs were obtained during admission in patients who were frequently acutely unwell and tachycardic, rather than under controlled conditions. As such, the accuracy of the coding is likely to have been reduced, particularly when evaluating the degree of
ST segment changes, and this was reflected in the comparatively low agreement between paper and electronically coded ECGs. However, myocardial ischaemia on the ECG is often transient and more likely to be present during the initial presentation, and therefore, we feel that the use of ECGs obtained during routine clinical assessments was more appropriate and less likely to underestimate the prevalence of myocardial ischaemia.

\section{Conclusion}

Raised troponin was found in $\sim 10 \%$ of patients admitted to hospital with acute exacerbation of COPD and the majority of these patients meet the Universal Definition of Myocardial Infarction. The proportion of patients in whom MI occurred as a consequence of plaque rupture and coronary thrombosis, or who would benefit from further cardiac investigation and secondary prevention is unknown.

\section{SUPPORT STATEMENT}

D.A. McAllister was supported by a Fellowship Grant from Chest, Heart and Stroke Scotland. N.L. Mills is supported by a British Heart Foundation Intermediate Clinical Research Fellowship (FS/10/024/ 28266).

\section{STATEMENT OF INTEREST}

Statements of interest for J.D. Maclay and W. MacNee can be found at www.erj.ersjournals.com/site/misc/statements.xhtml

\section{ACKNOWLEDGEMENTS}

The authors wish to thank D. Maxwell and S-J. Cheuk (University of Edinburgh, Edinburgh, UK) for assisting with this study.

\section{REFERENCES}

1 McGarvey LP, John M, Anderson JA, et al. Ascertainment of causespecific mortality in COPD: operations of the TORCH Clinical Endpoint Committee. Thorax 2007; 62: 411-415.

2 Anthonisen NR, Connett JE, Enright PL, et al. Hospitalizations and mortality in the Lung Health Study. Am J Respir Crit Care Med 2002; 166: 333-339.

3 Hole DJ, Watt GCM, Davey-Smith G, et al. Impaired lung function and mortality risk in men and women: findings from the Renfrew and Paisley prospective population study. BMJ 1996; 313: 711-715.

4 Huiart L, Ernst P, Ranouil X, et al. Oral corticosteroid use and the risk of acute myocardial infarction in chronic obstructive pulmonary disease. Can Respir J 2006; 13: 134-138.

5 Donaldson GC, Hurst JR, Smith CJ, et al. Increased risk of myocardial infarction and stroke following exacerbation of COPD. Chest 2010; 137: 1091-1097.

6 Baillard C, Boussarsar M, Fosse J-P, et al. Cardiac troponin I in patients with severe exacerbation of chronic obstructive pulmonary disease. Intensive Care Med 2003; 29: 584-589.

7 Brekke PH, Omland T, Holmedal SH, et al. Troponin T elevation and long-term mortality after chronic obstructive pulmonary disease exacerbation. Eur Respir J 2008; 31: 563-570.

8 Chang CL, Robinson SC, Mills GD, et al. Biochemical markers of cardiac dysfunction predict mortality in acute exacerbations of COPD. Thorax 2011; 66: 764-768.

9 Canto JG, Shlipak MG, Rogers WJ, et al. Prevalence, clinical characteristics, and mortality among patients with myocardial infarction presenting without chest pain. JAMA 2000; 283: 32233229.

10 Collinson PO, Premachandram S, Hashemi K, et al. Prospective audit of incidence of prognostically important myocardial damage in patients discharged from emergency department. BMJ 2000; 320: $1702-1705$. 
11 Thygesen K, Alpert JS, White HD, et al. Universal Definition of Myocardial Infarction. J Am Coll Cardiol 2007; 50: 2173-2195.

12 Apple FS, Wu AH, Jaffe AS. European Society of Cardiology and American College of Cardiology guidelines for redefinition of myocardial infarction: how to use existing assays clinically and for clinical trials. Am Heart J 2002; 144: 981-986.

13 Prineas RJ. The Minnesota Code Manual of Electrocardiographic Findings: Standards and Procedures for Measurement and Classification. Boston, J Wright, 1982.

14 Macfarlane PW, Latif S. Automated serial ECG comparison based on the Minnesota code. J Electrocardiol 1996; 29: Suppl. 1, 29-34.

15 Swap CJ, Nagurney JT. Value and limitations of chest pain history in the evaluation of patients with suspected acute coronary syndromes. JAMA 2005; 294: 2623-2629.

16 Anthonisen NR, Manfreda J, Warren CP, et al. Antibiotic therapy in exacerbations of chronic obstructive pulmonary disease. Ann Intern Med 1987; 106: 196-204.

17 Expert Panel on Detection, Evaluation, and Treatment of High Blood Cholesterol in Adults (Adult Treatment Panel III). Executive Summary of the Third Report of the National Cholesterol Education Program (NCEP). JAMA. 2001; 285: 2486-2497.

18 Brekke $\mathrm{PH}$, Omland $\mathrm{T}$, Holmedal $\mathrm{SH}$, et al. Determinants of cardiac troponin $\mathrm{T}$ elevation in COPD exacerbation - a crosssectional study. BMC Pulm Med 2009; 9: 35.

19 Lualdi JC, Goldhaber SZ. Right ventricular dysfunction after acute pulmonary embolism: pathophysiologic factors, detection, and therapeutic implications. Am Heart J 1995; 130: 1276-1282.

20 Sims MW, Margolis DJ, Localio AR, et al. Impact of pulmonary artery pressure on exercise function in severe COPD. Chest 2009; 136: 412-419.

21 Abraham AS, Cole RB, Green ID, et al. Factors contributing to the reversible pulmonary hypertension of patients with acute respiratory failure studies by serial observations during recovery. Circ Res 1969; 24: 51-60.

22 Dransfield MT, Rowe SM, Johnson JE, et al. Use of $\beta$ blockers and the risk of death in hospitalised patients with acute exacerbations of COPD. Thorax 2008; 63: 301-305.

23 van Gestel YR, Hoeks SE, Sin DD, et al. Impact of cardioselective $\beta$-blockers on mortality in patients with chronic obstructive pulmonary disease and atherosclerosis. Am J Respir Crit Care Med 2008; 178: 695-700.

24 Short PM, Lipworth SIW, Elder DHJ, et al. Effect of $\beta$ blockers in treatment of chronic obstructive pulmonary disease: a retrospective cohort study. BMJ 2011; 342: d2549-d2549.

25 Libby P. Inflammation in atherosclerosis. Nature 2002; 420: 868-874.

26 Wedzicha JA, Seemungal TA, MacCallum PK, et al. Acute exacerbations of chronic obstructive pulmonary disease are accompanied by elevations of plasma fibrinogen and serum IL-6 levels. Thromb Haemost 2000; 84: 210-215.

27 Hurst JR, Perera WR, Wilkinson TMA, et al. Systemic and upper and lower airway inflammation at exacerbation of chronic obstructive pulmonary disease. Am J Respir Crit Care Med 2006; 173: 71-78.

28 Maclay JD, McAllister D, Johnston S, et al. Increased platelet activation in stable and exacerbation of COPD. Thorax 2011; 66 769-774.

29 Sabatine MS, Morrow DA, de Lemos JA, et al. Detection of acute changes in circulating troponin in the setting of transient stress test-induced myocardial ischaemia using an ultrasensitive assay: results from TIMI 35. Eur Heart J 2009; 30: 162-169.

30 National Institute for Health and Clinical Excellence. Chest pain of recent onset. http://guidance.nice.org.uk/CG95 Date last accessed: April 13, 2010. Date last updated: January 19, 2012.

31 Brekke $\mathrm{PH}$, Omland $\mathrm{T}$, Smith $\mathrm{P}$, et al. Underdiagnosis of myocardial infarction in COPD - Cardiac Infarction Injury Score (CIIS) in patients hospitalised for COPD exacerbation. Respir Med 2008; 102: 1243-1247.

32 Sin DD, Man SFP. Why are patients with chronic obstructive pulmonary disease at increased risk of cardiovascular diseases? The potential role of systemic inflammation in chronic obstructive pulmonary disease. Circulation 2003; 107: 1514-1519.

33 Buckingham R, Lowe D, Roberts M, et al. National COPD Audit 2008. London, Royal College of Physicians, 2008.

34 Mahajan N, Mehta Y, Rose M, et al. Elevated troponin level is not synonymous with myocardial infarction. Int J Cardiol 2006; 111: 442-449.

35 Rutschmann OT, Cornuz J, Poletti P-A, et al. Should pulmonary embolism be suspected in exacerbation of chronic obstructive pulmonary disease? Thorax 2007; 62: 121-125. 